\title{
Approximate Analytic Solution for the Spatiotemporal Evolution of Wave Packets undergoing Arbitrary Dispersion
}

\author{
Borge Nodland \\ Department of Physics and Astronomy, and Rochester Theory Center for Optical Science and \\ Engineering, University of Rochester, Rochester, New York 14627
}

(Published in Physical Review E 55, 3647 (1997))

\begin{abstract}
We apply expansion methods to obtain an approximate expression in terms of elementary functions for the space and time dependence of wave packets in a dispersive medium. The specific application to pulses in a cold plasma is considered in detail, and the explicit analytic formula that results is provided. When certain general initial conditions are satisfied, these expressions describe the packet evolution quite well. We conclude by employing the method to exhibit aspects of dispersive pulse propagation in a cold plasma, and suggest how predicted and experimental effects may be compared to improve the theoretical description of a medium's dispersive properties.
\end{abstract}

PACS numbers: 03.40.Kf, 42.25.Bs, 52.35.Hr

Typeset using REVTEX 


\section{INTRODUCTION}

A systematic study of the propagation of electromagnetic pulses in a dispersive medium originated with Sommerfeld [1,2] and Brillouin [3, [4]. The subject has subsequently been studied by means of a number of interesting methods and novel interpretations; see for

example references [5 8] and references therein. Some of these methods are extensions to the classic Sommerfeld-Brillouin theory.

The purpose of this paper is firstly to provide an analytic expression for the behavior of dispersive wave packets by use of a straightforward method [see Eq. (8)], along with a description of the initial conditions that must be satisfied in order to render this expression valid. Secondly, we furnish the explicit formula that results when the method is applied to the case of Gaussian pulses in a cold plasma [see Eq. (16)]. We conclude by using the method to discuss and exhibit features of the dispersive propagation and interaction of multiple pulses in such a plasma.

\section{GENERAL METHOD}

The method we employ is based on Taylor expansion techniques and Gaussian wave packet expansion techniques that allow required integrals to be computed exactly. More specifically, we expand an initial wave packet as a superposition of Gaussian packets; then power-expand the dispersion relation to second order around the dominant wavenumber of each Gaussian. This allows for an exact solution of the Fourier integral describing the evolution of the packet.

We take as our starting point the standard linear Fourier integral [9, 10] describing the propagation of a pulse $E(x, t)$ in a medium of dispersion $\omega(k)$ :

$$
E(x, t)=\int_{-\infty}^{\infty} A(k) e^{i(k x-\omega(k) t)} d k
$$

where $A(k)$ must be chosen to satisfy the initial boundary conditions for $E(x, t)$ [9]. For simplicity, we assume that $\frac{d}{d t} E(x, 0)=0$, so that $A(k)$ is just the Fourier transform of the 
initial pulse $E(x, 0)=E_{0}(x)$ [9, 10,

$$
A(k)=\frac{1}{2 \pi} \int_{-\infty}^{\infty} E_{0}(x) e^{-i k x} d x
$$

We then take the real part of Eq. (1), assume $A(k)$ is real, and denote $\operatorname{Re} E(x, t)$ by $E(x, t)$ for simplicity

$$
E(x, t)=\int_{-\infty}^{\infty} A(k) \cos (k x-\omega(k) t) d k
$$

The next step is to find a fit to $E_{0}(x)$ in the form of a superposition of sinusoidal wave packets with a Gaussian envelope

$$
E_{0}(x)=\sum_{i=1}^{n} f_{i} e^{-a_{i} x^{2}} \cos \left(b_{i} x\right)
$$

where the constants $a_{i}, b_{i}, f_{i}$ are real, and $a_{i}>0$. Such a fit can normally be found for a localized initial packet $E_{0}(x)$. The Fourier transform of Eq. (四) can then be found exactly as

$$
A(k)=\frac{1}{2 \pi} \int_{-\infty}^{\infty} E_{0}(x) e^{-i k x} d x=\frac{1}{4 \sqrt{\pi}} \sum_{i=1}^{n} \frac{f_{i}}{\sqrt{a_{i}}}\left[e^{\left(\frac{-1}{4 a_{i}}\right)\left(k-b_{i}\right)^{2}}+e^{\left(\frac{-1}{4 a_{i}}\right)\left(k+b_{i}\right)^{2}}\right] .
$$

This amplitude is real, so the assumption below Eq. (2) is valid. The dominant wave numbers in Eq. (5) are $\pm b_{i}, i=1, n$, so we expand the dispersion relation $\omega(k)$ to second order around each point $\pm b_{i}, i=1, n$, separately:

$$
\omega\left(k, \pm b_{i}\right)=s_{0}\left( \pm b_{i}\right)+k s_{1}\left( \pm b_{i}\right)+k^{2} s_{2}\left( \pm b_{i}\right)
$$

Equation (6) constitutes $2 n$ expansions of $\omega(k)$, each expansion being centered around one of the $2 n$ points $\pm b_{i}, i=1, n$. The coefficients $s_{j}\left( \pm b_{i}\right)$ will in general depend on the parameters describing the dispersion relation $\omega(k)$ of the dispersive medium.

We can now obtain a good approximation to Eq. (3) by using Eqs. (5) and (6) to expand the integrand in Eq. (3) as a sum of $2 n$ terms, where each term is centered on the major wave numbers $\pm b_{i}, i=1, n$ of the initial packet: 


$$
\begin{aligned}
E(x, t) & =\frac{1}{4 \sqrt{\pi}} \sum_{i=1}^{n} \frac{f_{i}}{\sqrt{a_{i}}}\left\{\int_{-\infty}^{\infty} e^{\left(\frac{-1}{4 a_{i}}\right)\left(k-b_{i}\right)^{2}} \cos \left[-t s_{0}\left(b_{i}\right)+k\left(x-t s_{1}\left(b_{i}\right)\right)-t k^{2} s_{2}\left(b_{i}\right)\right] d k\right. \\
& \left.+\left(b_{i} \rightarrow-b_{i}\right)\right\}
\end{aligned}
$$

Note that Eq. (7) arises from a generalized expansion approach to Eq. (3), since it incorporates an arbitrary number of different expansion points $\pm b_{i}$. The integrand in Eq. (3) is expanded to a sum of $2 n$ Gaussian terms by substituting Eq. (5) into Eq. (3), and the only modification of a term occurs via a Taylor expansion (6) of the factor $\omega(k)$ around the point where the term is most influential. The advantage of the expansion (7) is that the resulting integrals can now be found analytically as

$$
\begin{aligned}
E(x, t)= & \frac{1}{4} \sum_{i=1}^{n} \frac{f_{i}}{\sqrt{a_{i}}}\left\{F^{\frac{1}{4}}\left(t, a_{i}, b_{i}\right)\right. \\
& \times \exp \left(F\left(t, a_{i}, b_{i}\right)\left(4 a_{i}\right)^{-1}\left[\left[x-t s_{1}\left(b_{i}\right)\right] t b_{i} s_{2}\left(b_{i}\right)-\frac{1}{4}\left[x-t s_{1}\left(b_{i}\right)\right]^{2}-t^{2} b_{i}^{2} s_{2}^{2}\left(b_{i}\right)\right]\right) \\
& \times \cos \left(F ( t , a _ { i } , b _ { i } ) \left[\left(4 a_{i}\right)^{-2}\left\{\left[x-t s_{1}\left(b_{i}\right)\right] b_{i}-t\left[b_{i}^{2} s_{2}\left(b_{i}\right)+s_{0}\left(b_{i}\right)\right]\right\}\right.\right. \\
+ & \left.\left.\frac{1}{4}\left[x-t s_{1}\left(b_{i}\right)\right]^{2} t s_{2}\left(b_{i}\right)-t^{3} s_{2}^{2}\left(b_{i}\right) s_{0}\left(b_{i}\right)\right]-\frac{1}{2} \tan ^{-1}\left[4 t a_{i} s_{2}\left(b_{i}\right)\right]\right) \\
+ & \left.\left(b_{i} \rightarrow-b_{i}\right)\right\},
\end{aligned}
$$

where $F\left(t, a_{i}, b_{i}\right)=\left[\left(4 a_{i}\right)^{-2}+t^{2} s_{2}^{2}\left(b_{i}\right)\right]^{-1}$. Equation (8) is useful in the sense that it is an explicit formula containing only elementary functions, and in the sense that it is quite general. All one needs to do to apply it to a specific situation is to compute the power series of the dispersion relation around the main wave numbers of the initial wave packet. The accuracy of the expression (8) will of course increase as the number of expansion points are increased. The formula provides for expansions of $\omega(k)$ up to second order, but one could also of course limit the order to unity by taking $s_{2}\left( \pm b_{i}\right)=0$ in Eq. (8).

We now discuss the initial conditions that must hold for Eq. (8) to be valid. From Eq. (5), we see that the amplitudes of the two terms of the integrand in the contribution

$$
\frac{1}{4 \sqrt{\pi}} \frac{f_{i}}{\sqrt{a_{i}}} \int_{-\infty}^{\infty}\left[e^{\left(\frac{-1}{4 a_{i}}\right)\left(k-b_{i}\right)^{2}}+e^{\left(\frac{-1}{4 a_{i}}\right)\left(k+b_{i}\right)^{2}}\right] \cos (k x-\omega(k) t) d k
$$


to $E(x, t)$ in Eq. (3) are maximal at the points $k=b_{i}$ and $k=-b_{i}$. The amplitudes decay exponentially as $k$ moves away from the points $\pm b_{i}$, and are $e^{-1}$ of their maximal value when $k$ is at a distance $2 \sqrt{a_{i}}$ from either point. At a distance $5 \sqrt{a_{i}}$, the amplitudes are $e^{-6.25} \approx 1.9 \times 10^{-3}$ of their maximal value, which is so small that beyond this distance one may consider the contribution (9) to be negligible. The $k$-intervals

$$
I\left(b_{i}\right)=\left[b_{i}-5 \sqrt{a_{i}}, b_{i}+5 \sqrt{a_{i}}\right], I\left(-b_{i}\right)=\left[-b_{i}-5 \sqrt{a_{i}},-b_{i}+5 \sqrt{a_{i}}\right]
$$

may therefore be taken as the only two areas of the $k$-line where the contribution (9) to (3) is appreciable.

In order for Eq. (8) to be applicable, one must require that a contribution (9) (for a specific $i$ ) is appreciable only within the two areas of the $k$-line where the two second order expansions $\omega\left(k, \pm b_{i}\right)$ of $\omega(k)$ (for the above $i$ ) in Eq. (6) are valid. The remainders $\mid \omega(k)-$ $\omega\left(k, b_{i}\right) \mid$ and $\left|\omega(k)-\omega\left(k,-b_{i}\right)\right|$ will in general be small over a certain interval containing $b_{i}$ and another interval containing $\left(-b_{i}\right)$, respectively. We denote the largest two such intervals over which $\left|\omega(k)-\omega\left(k, b_{i}\right)\right|$ and $\left|\omega(k)-\omega\left(k,-b_{i}\right)\right|$ are small by $I^{\prime}\left(b_{i}\right)$ and $I^{\prime}\left(-b_{i}\right)$ respectively. These intervals depend on $\omega(k)$ and $\pm b_{i}$ [The remainder terms depend on the third derivative of $\omega(k)$, as is well known from Taylor expansion theory]. The requirement for Eq. (8) to be valid can accordingly be stated as

$$
I\left(b_{i}\right) \subset I^{\prime}\left(b_{i}\right), I\left(-b_{i}\right) \subset I^{\prime}\left(-b_{i}\right), \quad(i=1, n) .
$$

Therefore, when the intervals $I^{\prime}\left(b_{i}\right)$ and $I^{\prime}\left(-b_{i}\right)$ have been determined from $\omega(k)$ and $\pm b_{i}$, the domain of validity of Eq. (8) can be stated as an upper limit on the pulse shape parameters $a_{i}$ in Eq. (田), as noted from Eq. (10). Note that the $n$ criteria in Eq. (11) are independent, since the resultant packet $E(x, t)$ in Eq. (3) is a linear combination of $n$ independent contributions of the form (9), as seen from Eq. (5). 


\section{COLD PLASMA APPLICATION}

We now consider the specific case of a pulse propagating in a cold plasma. For simplicity, we assume that the initial pulse can be approximated well by a single normalized Gaussian packet centered at $x=0$,

$$
E_{0}(x)=e^{-a x^{2}} \cos (b x) .
$$

In this case, Eq. (8) must be applied with the parameters

$$
n=1, a_{i}=a, b_{i}=b, f_{i}=1 .
$$

For the cold plasma, we use the dispersion relation [1]

$$
\omega(k)=\sqrt{c^{2} k^{2}+\omega_{p}^{2}},
$$

where $c$ is the speed of light in vacuum, and $\omega_{p}$ is the plasma frequency.

We then expand Eq. (14) around $b$ and $-b$, to obtain the coefficients in Eq. (6) as

$$
\begin{aligned}
s_{0}(b) & =\frac{3}{2}\left(c^{2} \omega_{p}^{2} b^{2}+2 \omega_{p}^{4}\right) B^{\frac{3}{2}}, s_{1}(b)=c^{4} b^{3} B^{\frac{3}{2}}, s_{2}(b)=\frac{1}{2} c^{2} \omega_{p}^{2} B^{\frac{3}{2}}, \\
s_{0}(-b) & =s_{0}(b), s_{1}(-b)=-s_{1}(b), s_{2}(-b)=s_{2}(b),
\end{aligned}
$$

where $B=\left(b^{2} c^{2}+\omega_{p}^{2}\right)^{-1}$. These coefficients are general, since they are functions of the initial pulse parameter $b$.

Substitution of Eqs. (13) and (15) in Eq. (8) yields

$$
\begin{aligned}
E(x, t) & =\frac{1}{4} a^{-\frac{1}{2}} T^{\frac{1}{4}} \exp \left\{\left(x-t b^{3} c^{4} B^{\frac{3}{2}}\right) t b c^{2} \omega_{p}^{2}(8 a)^{-1} B^{\frac{3}{2}}-\left(x-t b^{3} c^{4} B^{\frac{3}{2}}\right)^{2}(16 a)^{-1} T\right. \\
& \left.-t^{2} b^{2} c^{4} \omega_{p}^{4}(16 a)^{-1} B^{3}\right\} \\
& \times \cos \left\{T \left[\frac{1}{16}\left(x-t b^{3} c^{4} B^{\frac{3}{2}}\right) a^{-2} b+\frac{1}{8}\left(x-t b^{3} c^{4} B^{\frac{3}{2}}\right)^{2} t c^{2} \omega_{p}^{2} B^{\frac{3}{2}}\right.\right. \\
& \left.-\frac{1}{16} t a^{-2} B^{\frac{3}{2}}\left(2 b^{2} c^{2} \omega_{p}^{2}+\omega_{p}^{4}\right)-\frac{1}{8} t^{3} \omega_{p}^{4} c^{4} B^{\frac{9}{2}}\left(3 b^{2} c^{2} \omega_{p}^{2}+2 \omega_{p}^{4}\right)\right] \\
& \left.-\frac{1}{2} \tan ^{-1}\left(2 t a c^{2} \omega_{p}^{2} B^{\frac{3}{2}}\right)\right\}+(b \rightarrow-b)
\end{aligned}
$$


where $T=\left(\frac{1}{16} a^{-2}+\frac{1}{4} t^{2} c^{4} \omega_{p}^{4} B^{3}\right)^{-1}$ and $B=\left(b^{2} c^{2}+\omega_{p}^{2}\right)^{-1}$. Equation (16) is a general equation, describing the dispersive behavior of pulses of the form (12) that propagate in a medium with dispersion of the form (14).

The intervals $I\left( \pm b_{i}\right)$ and the validity criteria for Eq. (16) are found by substitution of Eq. (13) in Eqs. (10) and (11). Since the dispersion relation $\omega(k)$ in Eq. (14) is symmetric about $k=0$, the validity criteria in Eq. (11) simplify. A second order expansion $\omega(k, b)$ of the square root $\omega(k)=\sqrt{c^{2} k^{2}+\omega_{p}^{2}}$ about a positive wave number $b$ approximates $\omega(k)$ quite well when $k>0$, but fails when $k<0$. One must therefore require the interval $I(b)$ in Eq. (10) to lie to the right of the origin of the $k$-line. According to Eq. (10), the left endpoint of $I(b)$ is to the right of the origin when $b>5 \sqrt{a}$. $I(b)$ then lies within $[0, b+5 \sqrt{a}]$, which is within the interval $I^{\prime}(b)$ of validity of the expansion $\omega(k, b)$ [given by Eqs. (6) and (15)] of $\omega(k)$ [given by Eq. (14)]. A similar result holds for the expansion $\omega(k,-b)$ of $\sqrt{c^{2} k^{2}+\omega_{p}^{2}}$ about a negative wave number $-b$. Hence, we have the useful initial condition that

$$
\frac{b}{\sqrt{a}}>5
$$

for Eq. (16) to be valid. In other words, the approximation (16) is accurate when the frequency $b$ of the initial pulse $E_{0}(x)$ in Eq. (12) is high, or when $E_{0}(x)$ is broad (i.e. when the pulse shape parameter $a$ is small). However, we see from Eq. (17) that short pulses are also well approximated by Eq. (16) when they are of high frequency, as well as low-frequency pulses of long duration.

In the specific illustrations and discussions below, the pulse parameters $a$ and $b$ were of course always chosen so that Eq. (17) was satisfied, with many choices such that $\frac{b}{\sqrt{a}}>>5$. As a double check, we also compared values for $E(x, t)$ given by Eq. (16) with the values for $E(x, t)$ obtained by numerically integrating Eq. (3) [using Eqs. (5), (12), (13) and (14)] for a systematic variation of values for $x, t, a, b, c$ and $\omega_{p}$. For values of $a$ and $b$ obeying Eq. (17), the agreement was excellent, as expected. The discrepancy between the exact and approximate values for $E(x, t)$ was typically found to be less than $10^{-4}$.

Equation (16) represents two pulses traveling in opposite directions, as we expect 10 
from the initial condition $\frac{d}{d t} E(x, 0)=0$, stated above Eq. (2). For simplicity, in the remaining part of this paper we focus only on the right-traveling pulse term in Eq. (16) (which is the first term in Eq. (16) when $b$ is positive).

We now outline some of the features of the dispersive behavior of a single pulse in a cold plasma, which can be observed from plots of Eq. (16). It is readily observed that the envelope of a short initial pulse broadens more rapidly as time elapses compared to the broadening of the envelope of a long initial pulse, the initial pulse frequencies being equal. This is of course well known, and can be explained analytically [10].

It is also seen that in general, the number of oscillations within the pulse envelope increases with time, and more interestingly, the effect of dispersion on the pulse is to shift highfrequency components of the pulse toward the spatial front of the pulse and low-frequency components of the pulse toward the spatial rear of the pulse (see the pulse E1 in Fig. (1)). This effect becomes more prominent as the initial pulse is shortened.

Additionally, the spatial packet velocity of a pulse increases as the pulse frequency is increased (with spatial packet velocity we mean $x_{\max } / t$, where $x_{\max }$ is the distance traveled by the spatial maximum of the pulse envelope during a given time period $t$. Temporal pulse velocity is similarly defined as $x / t_{\max }$, where $t_{\max }$ is the time that elapses before the temporal maximum of the pulse envelope appears at a given distance $x$ [5]). However, as expected, in no cases is the center of a packet beyond the point $x=c t$ at time $t$ if it is at the origin $x=0$ at time $t=0$. Here $c$ is the velocity of light in vacuum, appearing in Eq. (16) from the dispersion relation (14).

Moreover, the envelope shape is better maintained for a high frequency initial pulse than for a low frequency initial pulse, the initial pulse durations being equal. Therefore, increasing the frequency of a short pulse will improve the preservation of its shape as it travels through a dispersive medium, as well as increase its packet speed. The propagation of short, high-frequency pulses is a topic of strong current interest [12, 13.

We next examine the the dispersive evolution of more than one (right-traveling) pulse 
through a cold plasma. One can readily do this by repetitive use of Eq. (16). For example, the propagation of two initial pulses $E_{01}(x)=e^{-a_{1} x^{2}} \cos \left(b_{1} x\right)$ and $E_{02}(x)=e^{-a_{2} x^{2}} \cos \left(b_{2} x\right)$ separated by an initial time delay $d$ is described by

$$
E(x, t)=E 1(x, t)+E 2(x, t)
$$

where $E 1(x, t)$ and $E 2(x, t)$ are obtained by substituting $\left(t \rightarrow t+d, a \rightarrow a_{1}, b \rightarrow b_{1}\right)$ and $\left(a \rightarrow a_{2}, b \rightarrow b_{2}\right)$ respectively, into Eq. (16). The first pulse $E 1$ is here $d$ time units ahead of the second pulse $E 2$ when $E 2$ passes the origin $x=0$ at the time $t=0$ (A description of the general dispersive behavior of $m$ initial pulses $E_{0 j}$ of the form (何 separated by initial time delays $d_{j}$ can analogously be obtained from Eq. (8) by the set of substitutions $t \rightarrow t+d_{j}$, $a_{i} \rightarrow a_{i j}, b_{i} \rightarrow b_{i j}$ for $i=1, n$ and $\left.j=1, m\right)$.

Figures 1 through 4 depict some features of dispersive double pulse propagation, as described by Eqs. (16) and (18). These figures also illustrate some of the properties of single pulse propagation mentioned above (note that the packets in the top plots of Figs. 11 and 3 at the initial time $t=0$ are identical to those given by Eq. (12), except that their amplitudes have half the magnitude of those in Eq. (12). This is because half of the energy of the initial packets is carried by the left-traveling parts of $E(x, t)$, which are omitted from all plots).

Figure 1 shows how two initially distinct packets of the same frequency and duration gradually overlap and interact with each other due to the dispersive stretching of the two packets as time elapses. Figure 2 shows the results of the interaction after a very long time. One can clearly see the emergence of constructive and destructive interference effects among the different spatial regions of the coalescing pulses.

In Fig. 3, a high frequency, short pulse is dispatched after the dispatch of a low frequency, long pulse. We see that the high frequency pulse eventually overtakes the low frequency pulse, in accord with the single-pulse feature that the packet velocity is greater for a highfrequency initial pulse than a low-frequency initial pulse. The middle and bottom plots show how the high frequency pulse interferes with the various spatial parts of the low frequency 
pulse. The bottom plot depicts an instant of constructive interference between the two pulses, where the total amplitude is larger than the separate amplitudes of either pulse. For longer times than those shown in the figure, the high frequency pulse eventually passes and leaves the other pulse.

As a final example of a usage of Eq. (16), one may employ this Eq. to determine parameters $a_{j}, b_{j}$, and initial time delays $d_{j},(j=i, m)$ of $m$ pulses such that they will all have the same width and completely overlap at a specified time $t$ while propagating in a plasma of dispersion characterized by $c, \omega_{p}$ and Eq. (14). Figure 4 shows a two-pulse version of this, with $t=1000$. We see that strong destructive and constructive interference effects occur in this case. A comparison of such predicted effects with those found experimentally in a specific medium could be used to determine the medium's dispersion relation $\omega(k)$.

\section{SUMMARY AND CONCLUSIONS}

We have provided an analytic expression describing the propagation of dispersive wave packets [Eq. (8)] provided the packets satisfy the applicability criteria (11). The expression is obtained by a clear-cut method, and can be used to study properties of the propagation process (for example pulse velocity and multiple pulse interference effects due to dispersion).

In the specific case of packets propagating in a cold plasma, we used Eq. (8) with two terms, and provided the explicit, analytic expression that results in this case [Eqs. (16) and (18)]. For initial pulses satisfying the condition (17), Eq. (16) gives an accurate description of single or multiple pulse propagation in a dispersive cold plasma.

As a final note, it is conceivable that the presence or non-presence of effects predicted by Eqs. (8) and (16) could be used to verify, improve the equations for, or determine a substance's dispersive properties. 


\section{ACKNOWLEDGMENTS}

This research was supported by NSF Grant No. PHY94-15583. The numerical part of the work was done at the Laboratory for Laser Energetics. 


\section{REFERENCES}

[1] A. Sommerfeld, Ann. Phys. 44, 177 (1914).

[2] A. Sommerfeld, Optics (Academic, New York, 1954).

[3] L. Brillouin, Ann. Phys. 44, 203 (1914).

[4] L. Brillouin, Wave Propagation and Group Velocity (Academic, New York, 1960).

[5] D. Anderson, J. Askne and M. Lisak, Phys. Rev. A 12, 1546 (1975).

[6] M. Tanaka, M. Fujiwara and H. Ikegami, Phys. Rev. A 34, 4851 (1986).

[7] L. Muschietti and C. T. Dunn, Phys. Fluids B 5, 1383 (1993).

[8] G. C. Sherman and K. E. Oughstun, J. Opt. Soc. Am. B 12, 229 (1995).

[9] G. B. Whitham, Linear and Nonlinear Waves (Wiley, New York, 1974), Chap. 11.

[10] J. D. Jackson, Classical Electrodynamics, 2nd ed., (Wiley, New York, 1975), Chap. 7.

[11] N. A. Krall and A. W. Trivelpiece, Principles of Plasma Physics, (San Francisco Press, San Francisco, 1986), p. 149.

[12] C. D. Decker et al., Phys. Plasmas, 3, 2047 (1996).

[13] M. D. Feit, J. C. Garrison and A. M. Rubenchik, Phys. Rev. E, 53, 1068 (1996). 


\section{FIGURES}

FIG. 1. Temporal evolution of two pulses E1 (right) and E2 (left) in a cold plasma, obtained from Eqs. (16) and (18). The initial time delay between the two packets is $d=1.5$. Both pulses are identical, with initial-value parameters $a_{1}=a_{2}=10$ and $b_{1}=b_{2}=20 \frac{\omega_{p}}{c}$. The plasma is characterized by $c=1$ and $\omega_{p}=1$. The spatial distribution of the total field $E(x, t)=E 1(x, t)+E 2(x, t)$ is shown at times $t=0$ (top), $t=500$ (middle) and $t=1000$ (bottom).

FIG. 2. Same as in Fig. 1, but for $t=5000$ (top) and $t=100000$ (bottom).

FIG. 3. Temporal evolution of two pulses $E 1$ (right) and $E 2$ (left) in a plasma, where $E 1$ is characterized by $a_{1}=5, b_{1}=20 \frac{\omega_{p}}{c}, E 2$ by $a_{2}=100, b_{2}=60 \frac{\omega_{p}}{c}$, and the plasma by $c=1, \omega_{p}=1$ and Eq. (14). $E 1$ is initially $d=1.1$ time units ahead of $E 2$. The spatial distribution of the total field $E(x, t)=E 1(x, t)+E 2(x, t)$ is shown at times $t=0$ (top), $t=500$ (middle) and $t=1000$ (bottom).

FIG. 4. Spatial dependence of a packet $E 1(x, t)$ (top) and a packet $E 2(x, t)$ (middle) after they have both traversed a cold plasma (characterized by $c=1, \omega_{p}=1$ and Eq. (14)) for a period of $t=1000$ time units. Initially, $E 1$ was $d=1.1$ time units ahead of $E 2 . \quad E 1$ is characterized by $a_{1}=10, b_{1}=20 \frac{\omega_{p}}{c}$ and $E 2$ by $a_{2}=2, b_{2}=40 \frac{\omega_{p}}{c}$. The bottom plot shows the total field $E(x, 1000)=E 1(x, 1000)+E 2(x, 1000)$. 
FIGURE 1
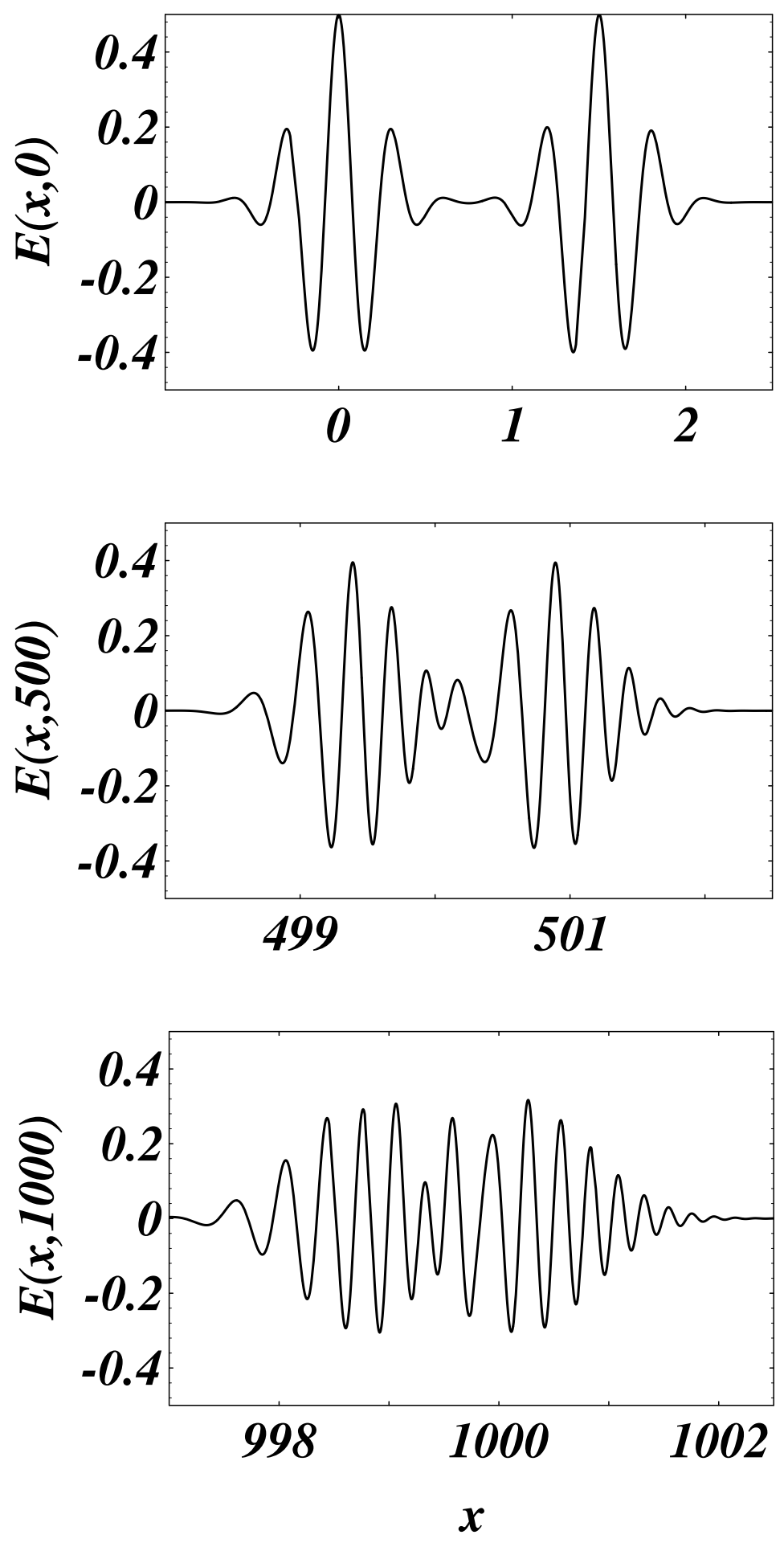
FIGURE 2
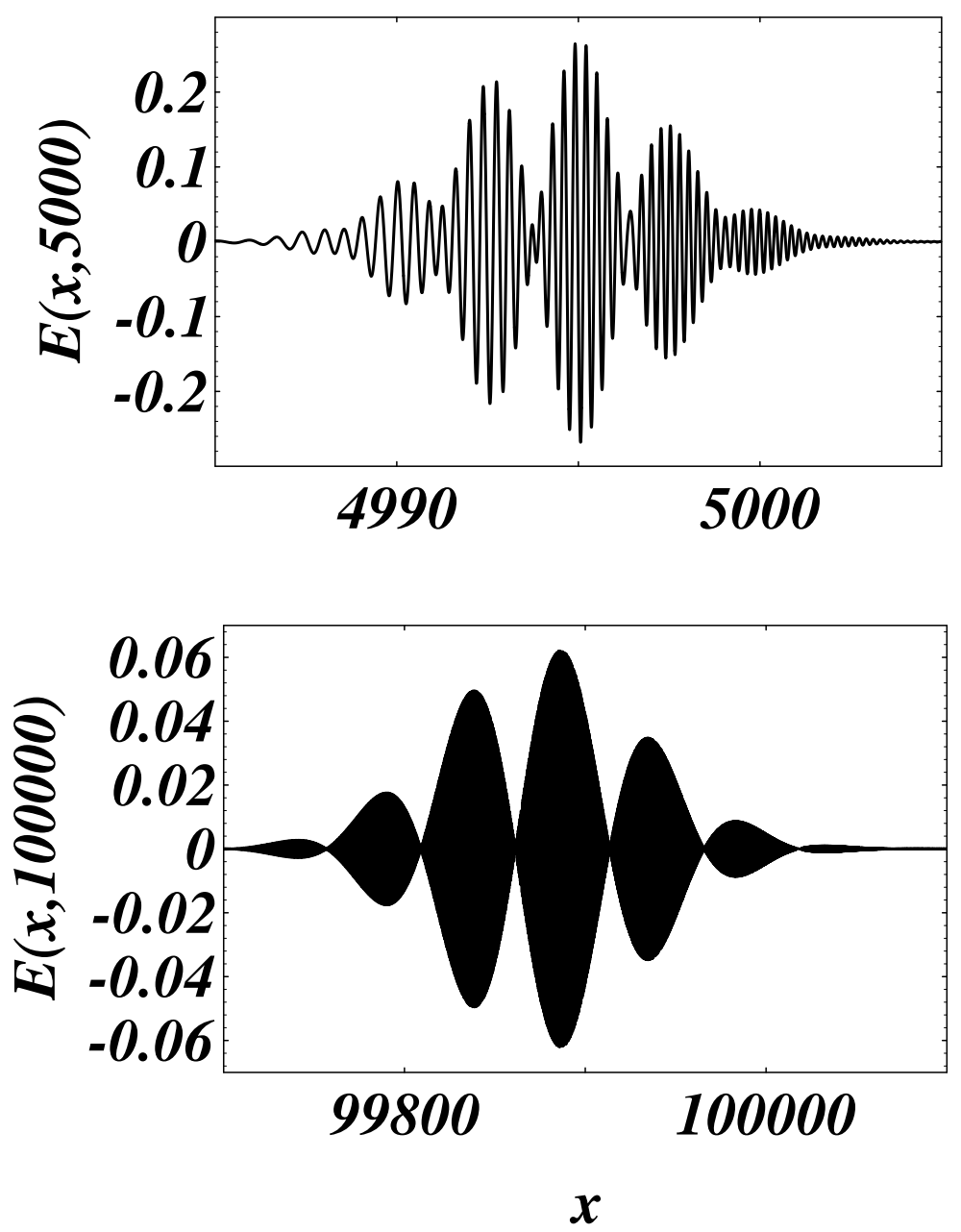
FIGURE 3
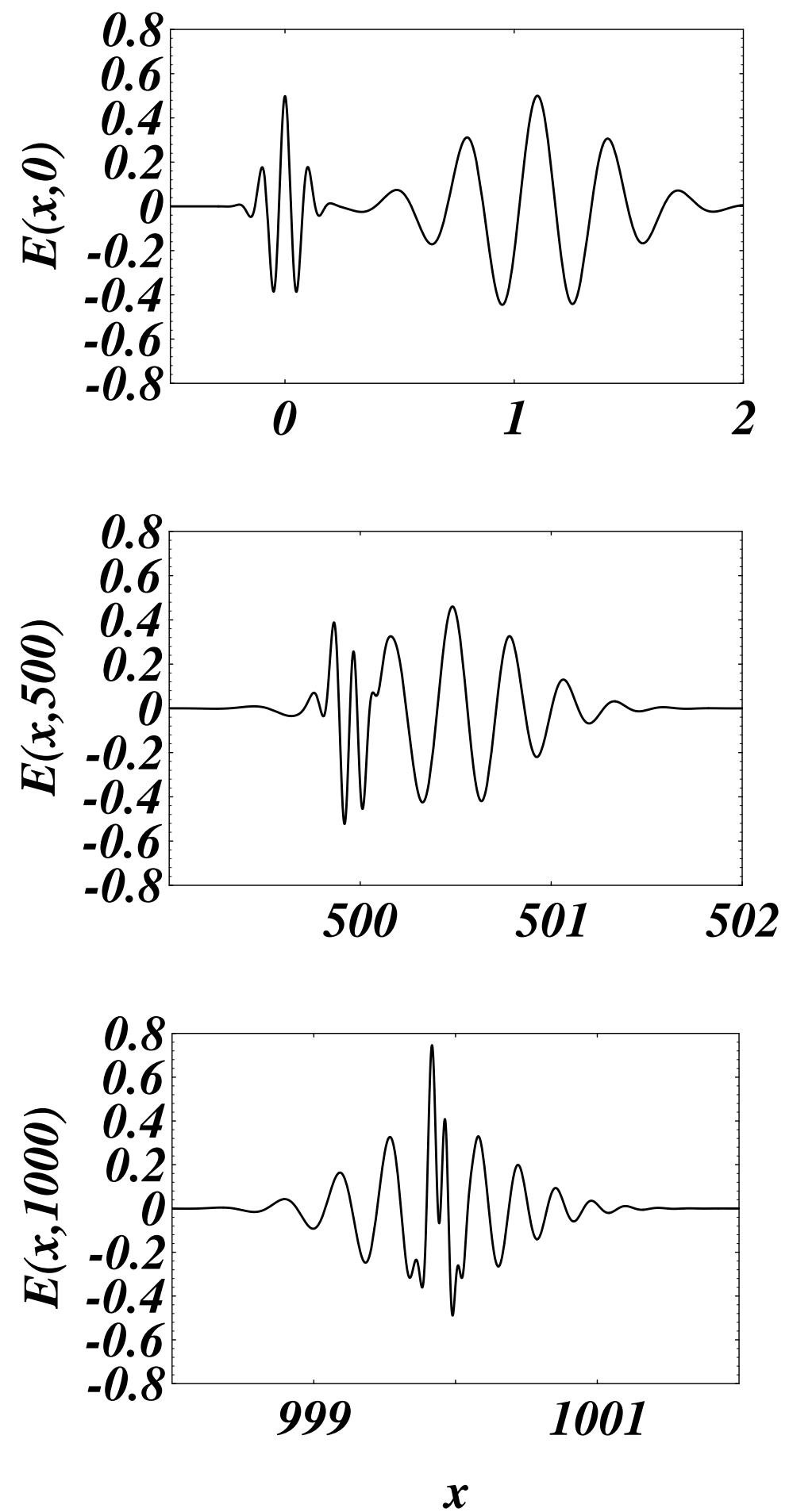
FIGURE 4
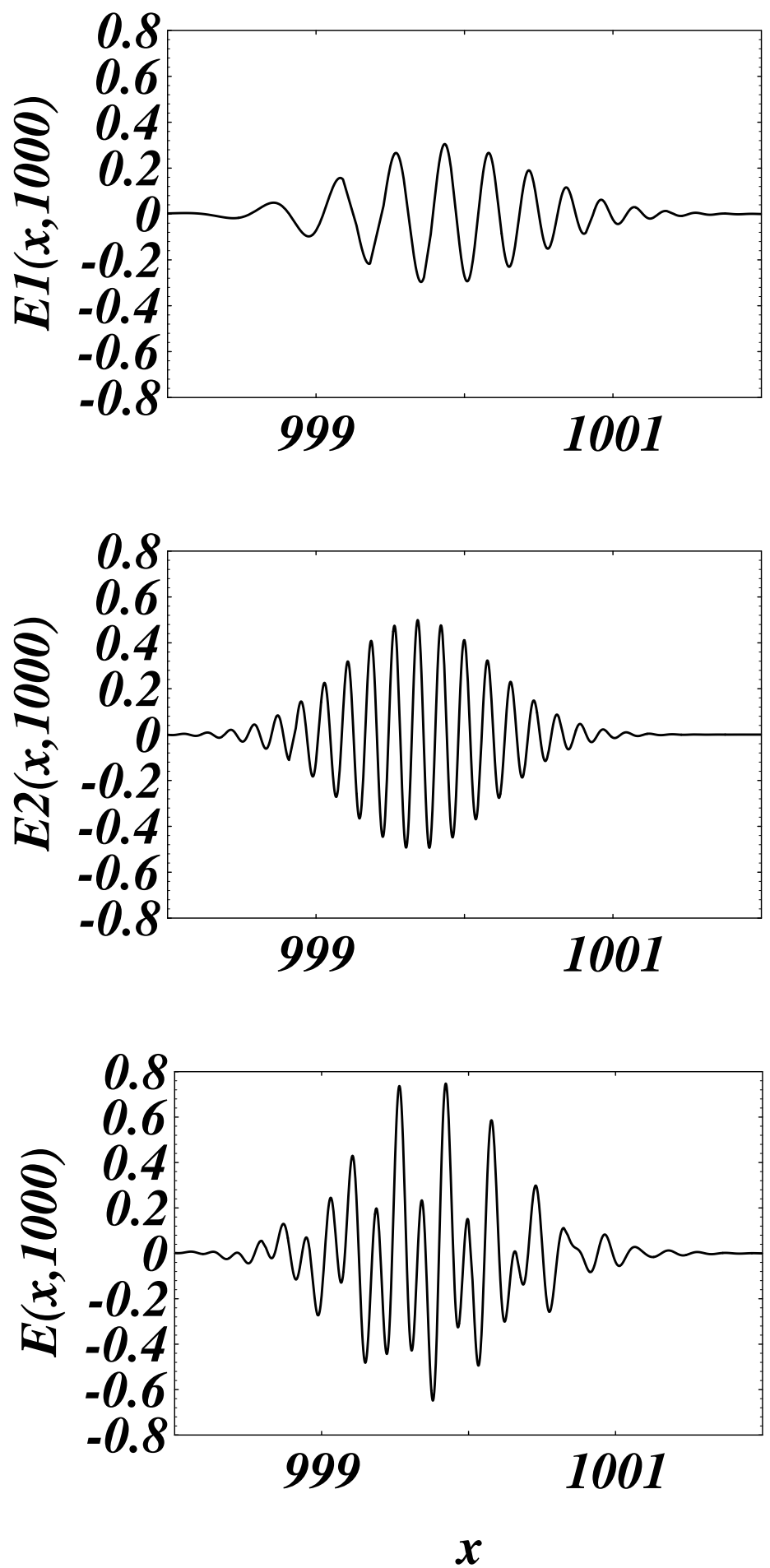